\title{
Análisis de requerimientos y diseño de modelo de datos para el desarrollo de una solución ERP vertical adaptada al sector cerámico
}

\author{
R.F. OLTRA-BADENES ${ }^{1}$, H. GIL-GÓMEZ ${ }^{1}$, R. BELLVER-LÓPEZ ${ }^{2}$, S. ASENSIO-CUESTA ${ }^{3}$ \\ 'Dpto. de Organización de Empresas. Universidad Politécnica de Valencia. Valencia, España \\ ${ }^{2}$ Dpto. de Informática de Sistemas y Computadores. Universidad Politécnica de Valencia. Valencia, España \\ ${ }^{3}$ Dpto. de Proyectos de Ingeniería. Universidad Politécnica de Valencia. Valencia, España
}

\begin{abstract}
En la actualidad, los sistemas de información existentes, y en concreto los ERP, no pueden dar un soporte adecuado a la gestión de las empresas de fabricación de baldosa cerámica, debido, entre otros motivos, a que no contemplan la existencia del tono, calibre y calidad dentro de un mismo producto. Esta particularidad, ocasionada por la falta de homogeneidad del producto (FHP), genera diversas problemáticas en la gestión del producto a través de los diferentes procesos de la empresa, como por ejemplo; la gestión de stocks, la gestión de pedidos de venta, la gestión de producción, etc. Así pues, se hace necesario el desarrollo de una solución ERP que sea capaz de gestionar el producto cerámico de forma adecuada, incluyendo tono, calibre y calidad. En este trabajo se analizan los requerimientos del sector cerámico, en cuanto a la identificación de los productos, y se propone un modelo de datos para dar respuesta a dichos requerimientos. El modelo se plantea como una guía básica para el desarrollo de soluciones ERP verticales adaptadas al sector cerámico.
\end{abstract}

Palabras clave: falta de homogeneidad en el producto, ERP, solución sectorial, solución vertical, sector cerámico.

Requirements analysis and data model design for the development of vertical ERP solutions for the ceramic industry

Currently, the existing information systems, and specifically the ERP, can not give adequate support to the management of manufacturing companies of ceramic tile, because, among other reasons, not to contemplate the existence of tone, size and quality within the same product. This feature, caused by the lack of homogeneity of the product (LHP), generates various problems in managing the product through the different business processes, such as, stocks management, order management, the production management, etc. Thus, it is necessary to develop an ERP solution that is able to manage adequately the ceramic product, including tone, size and quality. In this paper we analyze the requirements of the ceramic sector, in terms of product identification, and propose a data model to meet these requirements. The model arises as a basic guide for the development of vertical ERP solutions tailored to the ceramic industry.

Keywords: lack of de homogeneity in the product, ERP, industry solution, vertical solution, ceramic industry.

\section{INTRODUCCIÓN}

En el entorno empresarial actual, los Sistemas de Información (SI) juegan un papel muy importante a la hora de optimizar y mejorar la gestión de las empresas (1). Dentro de los SI, en los últimos años, los Enterprise Resources Planning (ERP) son las soluciones de referencia para empresas de todo el mundo (2). Los ERP permiten alcanzar la eficiencia corporativa y proporcionan beneficios en la coordinación, la comunicación y la gestión de procesos de la organización. Además, este tipo de SI permiten adaptar los entornos de trabajo, así como apoyar a la toma de decisiones en tiempo real (3), gestionar de forma adecuada su cadena de suministro (Supply Chain Management (SCM)), las relaciones con los clientes (Customer Relationship Management (CRM)), las relaciones con los proveedores (Supply Relationship Management (SRM)), y el e-business (4). La gestión adecuada de dichas relaciones de las empresas con su entorno resulta sumamente importante, hasta el punto de ser estratégicas y percibirse como un activo de estas (5), (6). Los ERP se presentan como sistemas capaces de realizar dicha gestión de forma adecuada (4).

Sin embargo, los ERP implantados en el sector cerámico español, no satisfacen adecuadamente los requerimientos específicos de dicho sector (7), (8), por lo que resultan ineficaces e ineficientes para gestionar los procesos de la empresa de forma adecuada (8). Esto es debido a que se trata de ERP genéricos, que no se adaptan a las características particulares del sector cerámico para resolver problemáticas importantes como la "Falta de Homogeneidad del Producto" (FHP). La FHP se define como "la carencia de la homogeneidad requerida por el cliente en los productos" y se debe a la imposibilidad de fabricar productos homogéneos, bien en un mismo lote o entre los distintos lotes obtenidos de un proceso (9). Esta particularidad del sector cerámico es un problema que tiene un gran impacto en el ámbito de la Dirección de Operaciones, sobre todo en las áreas de fabricación, 
planificación, gestión de pedidos y gestión de inventario, en las que surgen requerimientos adicionales y específicos de gestión del producto (9).

Para reducir el impacto de la FHP en la Dirección de Operaciones, se pueden proponer mejoras en la gestión de los procesos, así como herramientas de ayuda a la toma de decisiones, que pueden mejorar y agilizar en gran medida las decisiones relativas a los procesos de gestión, teniendo en cuenta la existencia de FHP. Existen trabajos de investigación en esa línea, basados en casos reales de aplicación en empresas líderes del sector cerámico, en los que se proponen modelos matemáticos, capaces de mejorar significativamente estos procesos de gestión (10).

Sin embargo, la aplicación de dichos modelos matemáticos, o de cualquier otra herramienta de ayuda a la toma de decisiones, no es posible sin un SI adecuado, capaz de soportar y recoger perfectamente los requisitos necesarios para la gestión óptima de los procesos en situaciones de FHP. Ante dicha necesidad, las soluciones sectoriales basadas en ERP estándar adecuadamente adaptadas a los requerimientos del sector se plantean como posibles soluciones.

La adaptación de un sistema ERP a un sector concreto, mediante el desarrollo de sus requerimientos particulares, se denomina solución "ERP vertical" o "sectorial", y en la actualidad, este tipo de soluciones lideran las tendencias de futuro en el desarrollo de SI para la gestión empresarial (11). Para desarrollar dichas soluciones son fundamentales los conocimientos específicos del sector y su problemática (12).

En el presente artículo, se analizan los requerimientos particulares del sector de fabricación de baldosa cerámica en cuanto a la identificación de producto con FHP. En base a dicho análisis se propone una metodología y un modelo de datos para el desarrollo de un SI adaptado al sector cerámico. El modelo planteado será válido tanto para el desarrollo de soluciones verticales basadas en ERP estándar, como para el desarrollo de software de gestión a medida.

El trabajo se estructura de la siguiente forma: en primer lugar se justifica la necesidad de un SI específico para el sector cerámico. A continuación se presentan las soluciones ERP verticales, como posible solución de dicha necesidad. Seguidamente se describen los requerimientos funcionales que genera la FHP en cuanto a la identificación de productos. Posteriormente se diseña un modelo de datos, para el desarrollo de una solución vertical a partir de ERP estándar. Finalmente, se extraen y presentan las conclusiones del trabajo realizado.

\section{NECESIDAD DE UN SISTEMA DE INFORMACIÓN ADAPTADO A LOS REQUERIMIENTOS DE LAS EMPRE- SAS DE FABRICACIÓN DE BALDOSAS CERÁMICAS}

La evolución histórica de los sistemas de información, comienza con la aparición de las primeras computadoras y los sistemas de gestión contable, y continúa con el desarrollo de los sistemas de gestión administrativa, los de control de stocks, los MRP (Material Requirement Planning), MRP II (Manufacturing Resources Planning), hasta llegar a los sistemas ERP Enterprise Resources Planning) (10). Estos sistemas, los ERP, integran todas las funciones y procesos de gestión de la empresa en una única aplicación, con lo que se logra una gestión eficaz y eficiente (14), y es por ello que este tipo de SI es la solución de referencia en el mercado actual.
Sin embargo, la implantación de sistemas ERP en el sector cerámico no resulta sencilla, ya que los ERP estándar deben ser modificados para satisfacer los requerimientos específicos (7), (8), entre los que destaca por su importancia la problemática de la diversificación y diferenciación de productos (8).

El problema de la diversificación y diferenciación de productos surge debido a la FHP existente en el producto final obtenido en la fase de producción. La FHP es una característica que se presenta en las empresas de fabricación de productos cerámicos, donde existen factores externos que afectan a la producción, tales como la humedad, la temperatura y el origen de las arcillas y los pigmentos. Las variaciones de estos factores imprevisibles e incontrolables provocan pequeñas diferencias en el color de los azulejos (diversidad de tonos) (7), la textura (15) y en los tamaños (diversidad de calibres)" (7). Sin embargo, a pesar de que en un mismo producto existan diferencias, es importante que al aplicar los azulejos en un revestimiento o pavimento, todos los productos sean de aspecto similar (mismo tono, calibre y calidad) (16).

Como consecuencia, la gestión adecuada de los distintos tonos, calibres y calidades existentes en un mismo producto, será decisiva para la satisfacción del cliente. Lo importante en este sentido será servir cada pedido de cliente con productos de un único tono, calibre y calidad, evitando de este modo posible problemas estéticos y funcionales en su aplicación: azulejos de un mismo color, pero con distintos tonos, que empeoran el acabado del conjunto de la pared o suelo, o diferentes calibres, que provocan desniveles o "escalones" en un mismo suelo o pared (9).

Esta circunstancia fuerza la necesidad de clasificar toda la producción en función de los valores de las tres características del producto: Tono, Calibre y Calidad (TCC). Por ello, en el proceso de fabricación resulta fundamental la fase de clasificación (7), (8), (17-19) donde todos los azulejos, uno por uno, son inspeccionados, de forma que los productos se clasifican y agrupan en función de su TCC, para su posterior embalado y paletizado. A partir dicha clasificación, los productos deben quedar identificados y dispuestos en el almacén con sus TCC perfectamente definidos, de manera que puedan gestionarse dichos productos en base a estas variables. Para soportar dicho proceso de clasificación y almacenamiento, se considera fundamental la existencia de un SI capaz de recoger y gestionar la información necesaria para el manejo de los diversos TCC de cada producto. Este SI además, deberá ser capaz de gestionar los diferentes procesos básicos de la empresa, como son la gestión de pedidos, la gestión de inventario, la planificación, la gestión de la producción o la previsión de la demanda, entre otros y dar soporte a la toma de decisiones. Evidentemente, el SI, para la gestión de estos procesos, debe tener en cuenta la clasificación de los productos y sus valores en cuanto a TCC.

Cabe añadir que, en el sector cerámico, la familia y el formato del artículo son también datos fundamentales, que deben estar presentes en el SI para una correcta gestión de los productos, pero que por lo general, no se encuentran en la mayoría de los ERP. Además de estos, hay otros datos que son importantes para una adecuada gestión de la información en el sector cerámico, como son el embalaje, peso o la posibilidad de tener diferentes marcas. Sin embargo, no se hace especial hincapié en ellos en el presente artículo, ya que la mayoría de los ERP líderes del mercado, ya incluyen estos campos y su gestión entre sus funcionalidades. 
Así pues, serán necesarios SI adecuados al sector cerámico, que resuelvan el problema que supone la FHP. Las empresas de dicho sector podrán optar por desarrollar SI a medida, o bien optar por la implantación de ERP estándar debidamente adaptados, también denominados ERP verticales o sectoriales. En ambos casos, será necesaria la definición de un modelo de datos que resuelva la problemática descrita.

\section{LOS ERP VERTICALES O SECTORIALES}

Los ERP son aplicaciones informáticas genéricas concebidas para soportar la gestión de los diferentes procesos de cualquier tipo de organización (20). Sin embargo, si se analizan en detalle, organizaciones de sectores industriales distintos, tienen procesos de negocio diferentes, que les son propios (12). Debido a ello, dependiendo del tipo de organización, los requerimientos del SI que deben utilizar pueden variar, bien sea debido a los productos, procesos, mercados o al tipo de gestión del sector en concreto. De este modo, la idea de SI genéricos con una filosofía horizontal o "one to fit all" ("uno que sirva para todos") (20), con la capacidad de acoplarse con éxito y servir para cualquier organización, presenta problemas en el momento de aplicarse y cubrir las necesidades de las organizaciones (21).

Para hacer frente a esta circunstancia, los fabricantes de ERP han adoptado una estrategia de "verticalización", en lo que parece ser una de las más evidentes tendencias estratégicas en los mercados de SI de empresa (1). Ésta puede definirse como "el ajuste, modificación o ampliación de una solución ERP en base a los procesos específicos de negocio de determinados sectores empresariales (22)".

Las soluciones ERP verticales proporcionan a las organizaciones SI más adecuados a su negocio, con un coste de implantación mucho menor que el de los desarrollos a medida (23). Como consecuencia, en los últimos años, los mayores fabricantes de ERP, se han dedicado al desarrollo de soluciones verticales, especializadas en diferentes sectores. Tal es el caso de los mayores fabricantes de software empresarial como IBM, Oracle, SAP o Microsoft (24). En la actualidad, los 60 mayores fabricantes de ERP, con sus soluciones verticales, cubren un total de 70 sectores empresariales (22), (24), como pueden ser, la industria de la moda (25), el sector de cerramientos de madera (26), el sector de los semiconductores (27), el entorno sanitario (28) o el de la Universidad (29).

Sin embargo, tras analizar la literatura existente y las soluciones ofrecidas por los fabricantes más importantes a nivel mundial, se observa que los verticales existentes hasta el momento, no cubren la problemática específica del sector cerámico (22), (24), lo cual deja patente una importante oportunidad de negocio que debería ser cubierta.

Ninguno de los ERP líderes del mercado (SAP, Microsoft, Oracle, IBM entre otros) son capaces de gestionar de forma adecuada la situación de FHP que se presenta en el sector cerámico.

Tampoco los ERP más recientes, como son algunos ERP de Software libre (OpenERP y OpenBravo por ejemplo) dan soluciones a esta problemática.

Aunque algún ERP, como es el caso de Dynamics AX de Microsoft (antes Axapta), posee campos identificativos como "talla" y "color", que podrían asimilarse de algún modo a "calibre" y "tono", estos campos son simplemente una etiqueta identificativa, por lo que no son operativos en una gestión adecuada a través de los procesos de Gestión de Operaciones descritos en (9) y (10). Es por ello, que en algunas de las empresas del sector cerámico que utilizan ERP, la gestión de calidades, tonos y calibres se lleva a través del campo “ $\mathrm{N}^{\mathrm{o}}$ de Lote". Mediante ese campo de la aplicación se identifica una combinación calidad-tono-calibre determinada. Esta solución, aunque mitiga en cierta medida la carencia de los ERP para gestionar la FHP, no es una identificación de calidad, tono y calibre, sino de un lote de producción, y por tanto, no es posible extraer información de una calidad, calibre o tono en concreto, sino tan solo del conjunto indivisible de los tres, lo que limita las opciones de tratamiento de la información. Por ejemplo, ante un pedido de venta de reposición de un cliente, que solicita una cantidad de un tono-calibre-calidad en concreto (el mismo que ya adquirió anteriormente, por ejemplo) en caso de que no quede inventario de ese mismo lote de producción, no es posible obtener información de qué cantidad hay en almacén que pudiera serle válida, de otro lote con similares características (mismo tono calibre y calidad) o incluso, por ejemplo, de unidades con el mismo tono y calidad, y un calibre similar, que podría satisfacer las necesidades del cliente.

Los ERP existentes, tampoco están preparados para gestionar la planificación, utilizando modelos y técnicas estadísticas adecuadas a la FHP, que puedan analizar producciones anteriores y sus resultados en cuanto a la FHP obtenida. Si estuvieran preparados con los campos indicados, podrían, por ejemplo, ajustar las previsiones, y con ello la planificación de producción, en base a los resultados obtenidos en diferentes épocas del año o en base al origen de las arcillas y pigmentos utilizados, que puedan ajustar en la mayor medida posible. Estos son factores que influyen en las calidades, tonos y calibres obtenidos, y que por tanto pueden ajustar en mayor medida las previsiones (9).

Estos son tan solo algunos de los aspectos que debería tener en cuenta un ERP vertical adaptado a la gestión del sector cerámico, pero que en la actualidad no se cubren por ninguno de los existentes.

Con el objetivo de servir de soporte al desarrollo de soluciones verticales para el sector cerámico, se analizan los requerimientos funcionales que un sistema ERP vertical para dicho sector debería implementar.

\section{REQUERIMIENTOS FUNCIONALES DE UNA EMPRE- SA DE FABRICACIÓN DE BALDOSAS CERÁMICAS}

El primer paso para desarrollar un ERP sectorial es hacer un análisis de requerimientos (30). Como ya se ha comentado anteriormente, uno de los principales problemas en los SI del sector cerámico es la falta de herramientas que permitan resolver adecuadamente los problemas actuales de diversificación y diferenciación de productos (8). Estos problemas están ocasionados por la FHP, que genera dificultades que afectan, en mayor o menor medida, a la gestión en diversas áreas y procesos de la empresa, como son la planificación de la producción, la previsión de ventas, la gestión de la producción, la gestión de costes, la gestión de pedidos de venta o la gestión de almacén.

Por ejemplo, en la planificación de la producción, a la hora de calcular las cantidades a fabricar de cada artículo hay que 
tener en cuenta que una misma orden de producción, de un artículo concreto y con un color determinado, puede dar lugar a distintos productos, debido a las variaciones en TCC. Por tanto, lo que se planifica, no es realmente lo que se obtiene, y puede variar sensiblemente, de forma que el producto fabricado puede no ser el requerido en la planificación, e incluso no ser adecuado para el destino que tenía inicialmente asignado. Por ello, es necesario tener estadísticas de las salidas reales de producción, y tener en cuenta estos datos (TCC) para la planificación de producción, de forma que la planificación se adecúe a las salidas de producción reales, con sus diferentes TCC.

Siguiendo con la gestión de la producción, hay que destacar que es necesario clasificar toda la producción en función del TCC obtenidos. Esto da lugar a que de una misma orden de producción pueden salir diferentes productos, dado que se obtienen diferentes cantidades de un artículo, con TCC distintos. Este hecho, que en principio parece trivial, ocasiona un grave problema, de gran complejidad de resolución en los SI. Ello es debido a que los SI para la gestión de la producción se basan en las Listas de Materiales, en las que diferentes inputs (materiales, manos de obra, maquinaria, etc.), generan un output (el producto fabricado). Sin embargo, en este caso, el proceso es al revés, y en la fase de clasificación de la producción, un input (el artículo planificado en la orden de producción) genera diferentes outputs (los diferentes productos que se obtiene finalmente de la orden de producción, con sus diferentes TCC). Esto rompe el concepto de producción que existe en los ERP, y en consecuencia se hace necesario cambiar este concepto, de forma que puedan adaptarse a esta problemática del proceso de producción cerámico.

Por otra parte, las cantidades de producto producidas, con sus correspondientes TCC debidamente clasificadas, deben entrar en almacén perfectamente definidas e identificadas, por lo que es necesario que dicha información esté presente en los procesos de gestión de stocks. De esta forma, cuando se reciba un pedido de venta de un cliente, se seleccionará la cantidad adecuada del artículo existente en stock, con el TCC requeridos en ese momento y por ese cliente en concreto, realizándose esta asignación de stock a pedido de forma óptima, tanto para el cliente como para la empresa fabricante de baldosa cerámica. Sin embargo, esta asignación de inventario a pedido es muy compleja en situaciones de FHP, como la que se presenta en el sector cerámico, y en muchas ocasiones, la asignación dista mucho de ser óptima, tal y como se recoge en (9).

Esta falta de optimización en la asignación de inventario a pedidos, puede ser corregida mediante el uso de herramientas de ayuda a la toma de decisiones, como son los modelos matemáticos. En (10) se presenta un estudio de un caso real de una empresa del sector cerámico, en el que se aplica un modelo matemático adaptado a la problemática de la FHP concreta del sector cerámico. Este modelo matemático, optimiza la asignación de inventario a pedidos, realizando una reasignación óptima del inventario existente a una cartera de pedidos real. Se demuestra que, en ese caso de estudio, la optimización puede llegar a incrementar el nivel de servicio en el corto plazo (mes siguiente) en un $17 \%$, y el de la facturación asociada en un $29 \%$ (10).

Sin embargo, para poder llevar a cabo la ejecución de este modelo matemático en la práctica, es necesario un sistema de información que sea capaz de recoger los datos, la información, de forma adecuada.
De este modo, las circunstancias que se han descrito derivadas de la FHP provocan que, para el desarrollo de ERP verticales para el sector cerámico, sea imprescindible el desarrollo de un modelo de datos que permita identificar de forma adecuada los productos, incluyendo las características derivadas de la FHP.

\section{DESARROLLO DE UN MODELO DE DATOS PARA LA CORRECTA IDENTIFICACIÓN DE PRODUCTOS EN EL SECTOR CERÁMICO}

Para solucionar los problemas que la FHP genera en los procesos de gestión de empresas, en primer lugar es necesario analizar las características particulares de los productos, para poder así proponer soluciones concretas.

En el sector cerámico, además de las variables TCC mencionadas a lo largo de este trabajo, hay otras características identificativas del producto (azulejo o baldosa cerámica), que deberían considerarse a la hora de desarrollar un ERP vertical, ya que son necesarias para la gestión de diferentes procesos. A continuación se presentan las características específicas necesarias para identificar correctamente un producto cerámico:

- Familia: esta característica define qué cometido va a tener el azulejo (suelo, pared, decoración de pared, etc.). Algunas de las familias más habituales suelen ser: pavimento, revestimiento, rodapié o cenefas.

- Formato: el formato define las dimensiones de longitud y anchura del azulejo. Se suele utilizar una nomenclatura del tipo "anchura x longitud".

- Modelo: es el diseño determinado que distingue a ese azulejo de los que son de su misma familia y con el mismo formato.

- Color: diferencia entre azulejos que, partiendo del mismo diseño, se han decorado con diferentes colores; es decir, podemos tener dos azulejos de la misma familia, con el mismo formato y que sean el mismo modelo, pero diferente color.

- Calidad: calidades según haya quedado el diseño impreso en el azulejo, la presencia de posibles deficiencias, etc.

- Calibre: el calibre permite distinguir entre azulejos que tienen exactamente las mismas características, pero que tienen un grosor distinto.

- Tono: el tono permite distinguir entre azulejos que tienen exactamente las mismas características, pero que tienen distinto tono del mismo color (más claro o más oscuro).

Dichas características deberán estar disponibles en una solución ERP vertical para el sector cerámico, de modo que permitan identificar correctamente cada uno de los productos, con el objetivo de realizar una gestión adecuada de los diferentes procesos empresariales en el ERP.

Sin embargo, es importante destacar que no en todos los procesos de la empresa es necesario identificar todas las características. Dependiendo del proceso empresarial, el artículo deberá identificarse con mayor o menor detalle.

Por ejemplo, en el momento en que se crea una orden de producción, aún no se sabe qué TCC se van a obtener como resultado del proceso de fabricación, por lo que es imposible identificar dichos valores en el momento de crear la orden de 
producción. Por ello, en el proceso de la creación de la orden de producción, los campos "Tono", "Calibre" y "Calidad" son innecesarios, y no deben aparecer. Pero en el momento en que se finaliza la producción, tras la fase de clasificación, cuando los productos acabados (baldosa cerámica) entran en el almacén, sí deben estar perfectamente identificados, y los campos de "Calidad", "Tono" y "Calibre", son campos Obligatorios.

Otro ejemplo se puede ver en el caso de la gestión de los pedidos de venta. En el momento de la creación del pedido, cuando se recibe del cliente, éste puede especificar un tono y calibre concreto (por ejemplo para que el producto sea igual al que recibió en una entrega anterior) o puede no especificarlos, porque le es indiferente el tono y el calibre. En este caso, los campos de Tono y Calibre, son opcionales. Posteriormente, cuando el pedido se entregue al cliente, sí debe quedar registrados los valores del tono y calibre entregados, por lo que dichos campos son obligatorios.

Similar es el caso de los campos Familia, Formato, Modelo o Color, que en el caso de la gestión comercial, por ejemplo en las tarifas de venta o descuentos o promociones pueden ser opcionales. Es decir, no es obligatorio marcar un descuento (por ejemplo un 3\%) para toda una familia, pero es una opción que se considera deseable. Puede ser deseable hacer una campaña de descuento para una familia en concreto en un momento dado. En ese caso, se utilizaría esa opción a la hora de generar el descuento. Pero también es posible que se quiera hacer un descuento en un modelo y formato concreto, o de un color (por ejemplo para retirarlo del stock y catálogo de productos) pero no de toda la familia a la que pertenece. En ese caso, el descuento se aplicaría utilizando los campos de modelo y formato, pero no el de familia, ya que si fuera así, se aplicaría a todos los modelos y formatos que pertenecen a ella. Por tanto, estos campos, en el proceso de aplicación de descuentos son opcionales, y se pueden utilizar o no, en función de a qué nivel de agrupación de producto se le quiera aplicar el descuento.

Por tanto, en cada proceso, e incluso dentro de un mismo proceso, los campos, Familia, Formato, Modelo, Color, Calidad, Tono y Calibre, pueden ser obligatorios, opcionales o innecesarios. A continuación se presenta una tabla en la que aparecen en las filas diferentes procesos de empresa y en las columnas los campos mencionados. En la intersección de fila y columna, se indica si el campo es obligatorio (x), opcional (o) o innecesario (i), en el proceso correspondiente.

La tabla 1 servirá de guía a analistas, diseñadores y desarrolladores de soluciones de software verticales para el sector cerámico. Como se puede observar, no se han incluido todas las áreas y procesos de la empresa, sino sólo los necesarios a tener en cuenta en el desarrollo de una solución vertical para el sector cerámico, en relación con la identificación de los productos. Por ello, no tiene sentido incluir, por ejemplo, el área de Recursos Humanos, ya que no tiene necesidades específicas en relación a la identificación de producto. Lo mismo sucede, por ejemplo, con el área de compras, que pese a estar relacionada con los productos, no tiene necesidades específicas de identificación de producto, ya que los requerimientos expuestos en este artículo surgen

TABLA 1. CAMPOS NECESARIOS PARA IDENTIFICAR LOS PRODUCTOS SEGÚN LOS PROCESOS
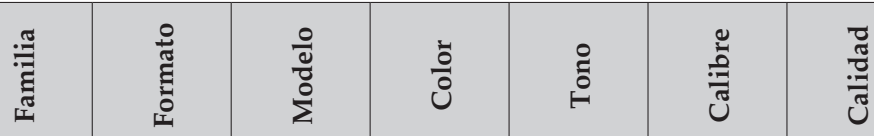

\begin{tabular}{|c|c|c|c|c|c|c|c|}
\hline \multicolumn{8}{|l|}{ Gestión comercial } \\
\hline Gestión de tarifas & o & $\mathrm{o}$ & $\mathrm{o}$ & o & $\mathrm{i}$ & $\mathrm{i}$ & $x$ \\
\hline Gestión de descuentos & o & $\mathrm{o}$ & $\mathrm{o}$ & o & $\mathrm{i}$ & $\mathrm{i}$ & $\mathrm{x}$ \\
\hline Campañas promocionales & o & $\mathrm{o}$ & $\mathrm{o}$ & o & $\mathrm{i}$ & $\mathrm{i}$ & $x$ \\
\hline \multicolumn{8}{|l|}{ Gestión de pedidos } \\
\hline Recepción, registro y creación del pedido & o & $\mathrm{x}$ & $\mathrm{X}$ & $x$ & $\mathrm{o}$ & o & $\mathrm{o}$ \\
\hline Cumplimentación del pedido & o & $x$ & $x$ & $x$ & $\mathrm{X}$ & $\mathrm{X}$ & $x$ \\
\hline Preparación, expedición y entrega & o & $x$ & $\mathrm{x}$ & $x$ & $\mathrm{X}$ & $\mathrm{x}$ & $\mathrm{X}$ \\
\hline Devoluciones & $\mathrm{o}$ & $x$ & $\mathrm{x}$ & $x$ & $x$ & $X$ & $x$ \\
\hline Planificación de la demanda & $\mathrm{X}$ & $X$ & $\mathrm{x}$ & $x$ & $\mathrm{o}$ & $\mathrm{o}$ & $x$ \\
\hline Planificación de la producción & $\mathrm{X}$ & $x$ & $x$ & $x$ & $\mathrm{o}$ & o & $\mathrm{x}$ \\
\hline \multicolumn{8}{|l|}{ Gestión de producción } \\
\hline Creación de la orden de producción & $\mathrm{o}$ & $X$ & $x$ & $x$ & $\mathrm{i}$ & $\mathrm{i}$ & $\mathrm{i}$ \\
\hline Finalización y cierre de la orden de producción & o & $x$ & $x$ & $x$ & $\mathrm{x}$ & $\mathrm{x}$ & $x$ \\
\hline Listas de materiales, rutas de producción, etc. & o & $\mathrm{X}$ & $\mathrm{X}$ & $x$ & $\mathrm{i}$ & $\mathrm{i}$ & $\mathrm{i}$ \\
\hline \multicolumn{8}{|l|}{ Gestión de almacén } \\
\hline Movimientos de almacén & $\mathrm{o}$ & $x$ & $x$ & $x$ & $X$ & $\mathrm{X}$ & $\mathrm{X}$ \\
\hline Recuentos de almacén & o & $X$ & $x$ & $x$ & $\mathrm{X}$ & $\mathrm{X}$ & $\mathrm{x}$ \\
\hline Gestión financiera & o & $X$ & $x$ & $x$ & $\mathrm{i}$ & $\mathrm{i}$ & $\mathrm{i}$ \\
\hline Gestión de costes & $\mathrm{X}$ & $\mathrm{X}$ & $\mathrm{x}$ & $\mathrm{x}$ & $\mathrm{i}$ & $\mathrm{i}$ & $x$ \\
\hline Diseño del producto & $\mathrm{X}$ & $\mathrm{x}$ & $\mathrm{x}$ & $\mathrm{x}$ & $\mathrm{i}$ & $\mathrm{i}$ & $\mathrm{i}$ \\
\hline
\end{tabular}


en la gestión del producto final, pero no en la gestión de las materias primas.

En el desarrollo de un ERP vertical, un factor clave es la definición de la base de datos y su estructura (30). El objetivo del diseño de base de datos es trasladar una situación, estructura o proceso del mundo real a un modelo de datos formal para ser implementado en un sistema de gestión de bases de datos determinado.

El diseño de una base de datos puede dividirse en los siguientes pasos (30):

1. Análisis de requerimientos: En este primer paso, se debe extraer, recoger y analizar toda la información necesaria para modelar correctamente la aplicación. Es necesario extraer la información del entorno real, de los futuros usuarios del sistema.

2. El diseño conceptual: se traducen los requerimientos funcionales del entorno real en un modelo conceptual (por ejemplo, el modelo entidad-relación (ER)) que se utiliza para describir el esquema conceptual.

3. El diseño lógico: se traduce el diseño conceptual en el modelo de datos (por ejemplo, el modelo relacional), apoyado por el sistema de gestión de base de datos en el que se va a implementar.

4. El diseño físico: se transforma el esquema lógico en un esquema físico adecuado para una configuración específica.

La fase de análisis de requerimientos, ha sido realizada en los apartados 4 y 5 . Por tanto, en el proceso de desarrollo de la Base de Datos para el desarrollo de un ERP vertical, la siguiente fase a abordar será el diseño conceptual.

\subsection{Diseño conceptual}

En el modelo conceptual se traducen los requerimientos funcionales del entorno real en un modelo conceptual (por ejemplo, el modelo ER). En este trabajo, se adopta una versión de la técnica de modelado Extended Entity Relation (EER), debido a su uso generalizado en el diseño de bases de datos relacionales (30).

Utilizando esta nomenclatura, se realiza un diagrama ER de la Base de Datos. Es importante destacar que, para simplificar el modelo conceptual con el objetivo de conseguir una fácil comprensión del mismo, no se desarrolla todo el modelo conceptual completo. Únicamente se desarrolla y explica la parte correspondiente a la identificación del producto, y su relación con la gestión de stocks y los pedidos de venta, como ejemplo de cómo obtener la solución deseada. De esta forma se presenta una metodología a seguir por parte de los desarrolladores de ERP para obtener un modelo conceptual completo. Así, los desarrolladores de ERP deberán desarrollar los modelos ER que incluyan el resto de procesos, de forma similar a la presentada en este artículo.

A continuación se muestra el diagrama ER propuesto.

Como puede observarse en el diagrama, un artículo se compondrá de los atributos estándar de cualquier ERP (código, nombre, descripción, etc.), más los atributos característicos del sector cerámico, como son formato, familia, color y modelo, siendo todos ellos obligatorios (restricción de existencia). Por tanto, cuando un artículo sea dado de alta en la base de datos, por ejemplo cuando aparece un nuevo diseño de azulejo, será necesaria la introducción de todos estos valores en el ERP, que irán relacionados con el artículo, y por tanto, estarán disponibles en el resto de procesos del SI.

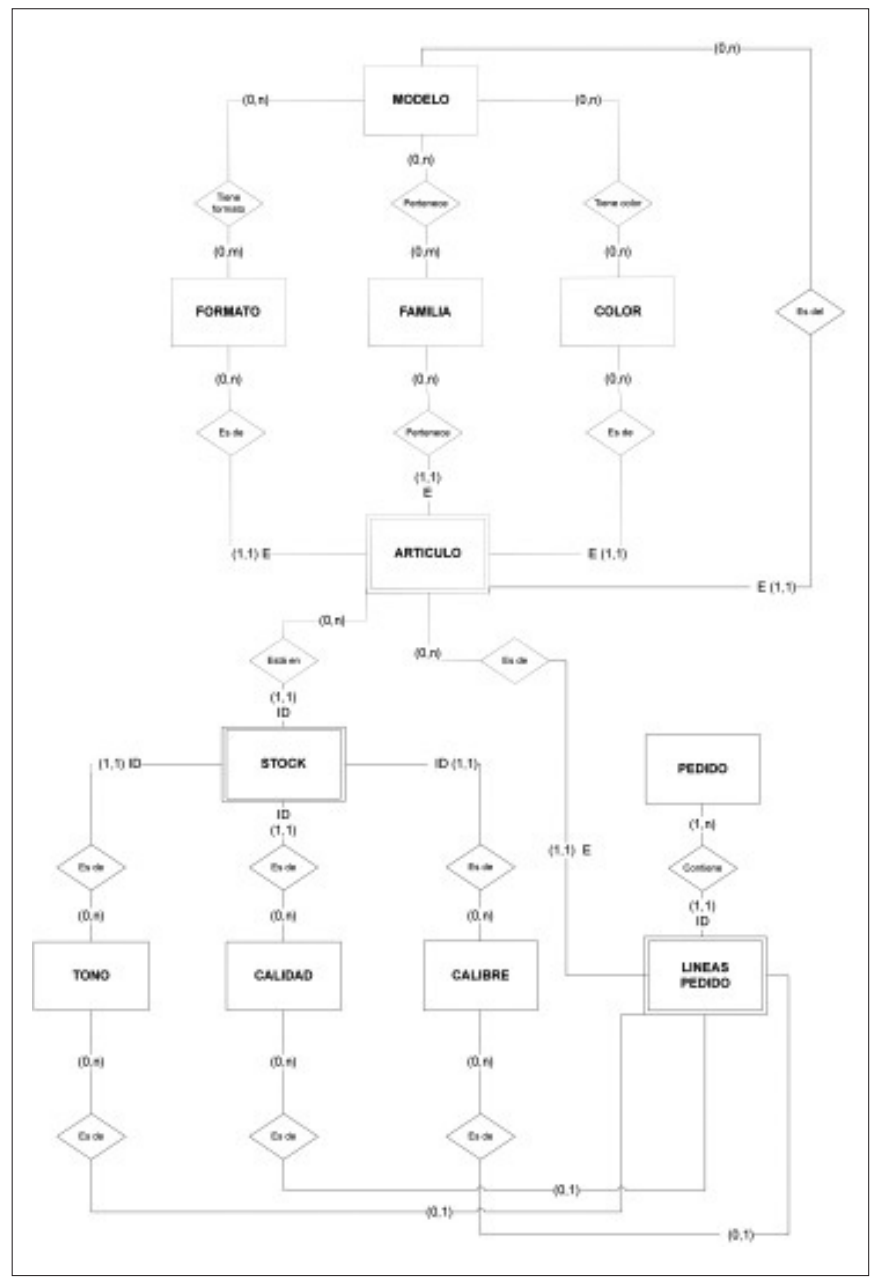

Figura 1. Diagrama Entidad-Relación para la Gestión de Stocks y Pedidos de Venta.

Por otra parte, según el diagrama ER descrito, un artículo disponible en stock, siempre deberá estar identificado por su código, calidad, tono y calibre (restricciones de identificación). Además, dado un artículo, se conocerá su familia, formato, color y modelo, y por tanto, en todo momento estarán identificados todos los campos necesarios para la identificación del artículo detallados en el apartado de requerimientos (apartado 4).

En cuanto a la gestión de pedidos, se observa que la línea de pedido debe incluir al artículo, y sus atributos de calidad, tono y calibre.

\subsection{Diseño lógico}

El siguiente paso en el desarrollo de la Base de Datos es definir la lógica de diseño, en la que se traduce el diseño conceptual en un modelo de datos concreto, para el sistema de gestión de base de datos en el que se decida realizar la implementación.

En el ejemplo propuesto, el modelo ER es implementado en el sistema de gestión de bases de datos "Microsoft Access". Evidentemente, en el desarrollo del ERP vertical los desarrolladores de la solución, deberán trasladar el modelo conceptual a la base de datos que utilice el ERP que se esté adaptando (Oracle, SQL, etc.) 


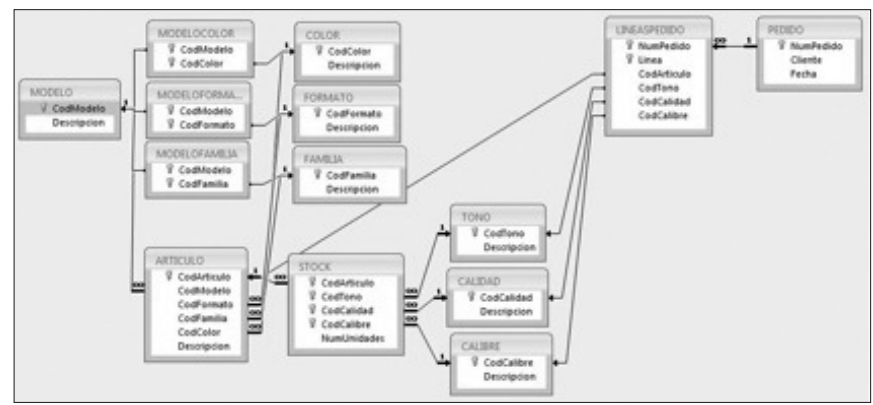

Figura 2. Diseño Lógico de la Base de Datos del ERP vertical en Microsoft Access.

En la Figura 2 se muestran las tablas y su relación, definidas a partir del diagrama ER desarrollado anteriormente. Es importante destacar que sólo se han incluido algunos campos de las diferentes tablas. Evidentemente, la tabla de artículos, o de pedidos, deben tener más campos que los mostrados, tales como el peso del artículo, su precio, etc., todos ellos existentes en los ERP estándar. La Figura 2 presenta únicamente los campos necesarios para la correcta identificación de los productos en el sector cerámico en base a los requerimientos descritos, si bien también se presentan algunos campos básicos de cada una de las tablas, necesarios para establecer las relaciones entre ellas. De nuevo cabe señalar que los desarrolladores de ERP deberán tener en cuenta tanto los campos propios de la problemática del sector cerámico como aquellos definidos en el ERP estándar, así como sus relaciones, al desarrollar la solución vertical.

La estructura de tablas definida, deberá ser implementada en el ERP vertical, obteniéndose una base de datos adaptada a la gestión de los productos en el sector cerámico.

Finalmente, a partir de la base de datos obtenida, se deberá realizar el diseño físico de la aplicación. Sin embargo, esta fase del desarrollo de la base de datos es totalmente dependiente del sistema a utilizar, de su lenguaje de desarrollo y del entorno de programación. Dicho diseño físico queda fuera del alcance del trabajo que se presenta.

Como aplicación práctica, y dentro de un proyecto de investigación que está actualmente en desarrollo por parte de los autores junto con una empresa del sector cerámico, se presenta la aplicación del modelo en uno de los ERP líderes del mercado, como es Dynamics AX.

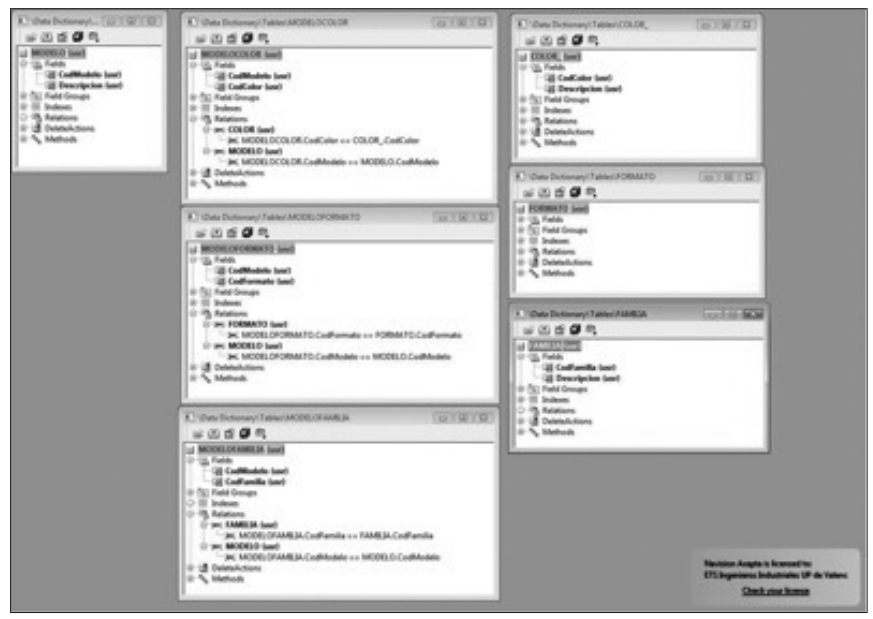

Figura 3. Tablas para la gestión del TCC en Dynamics Ax.
En la Figura 3 se muestran las tablas que se han creado en el ERP, necesarias para la gestión de la FHP a través del Tono, Calibre y Calidad en el sector cerámico.

En la Figura 4 se muestra el diseño de dichas tablas y los campos que contienen.

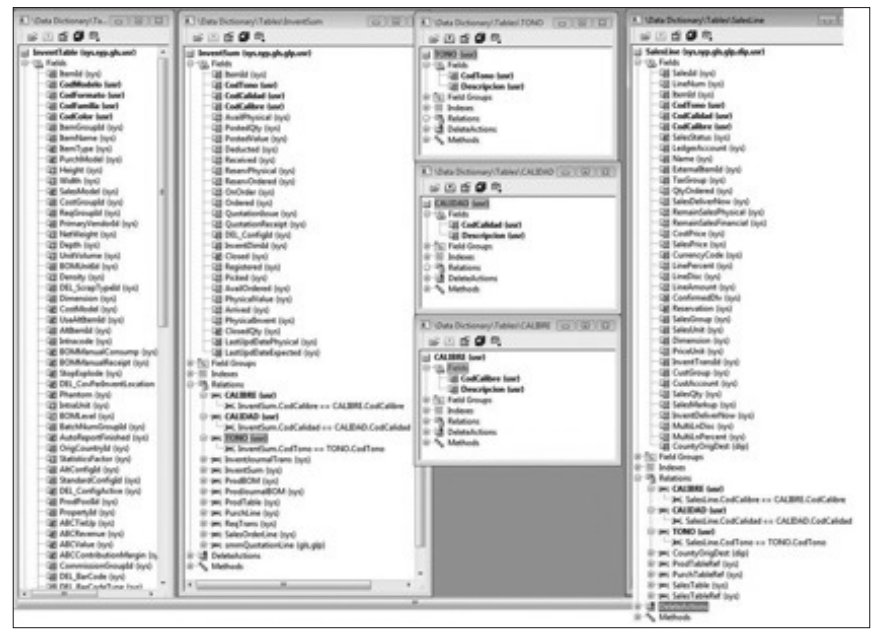

Figura 4. Diseño de tablas y sus relaciones para la gestión del TCC en Dynamics Ax.

En la Figura 5 y Figura 6 se muestran respectivamente los formularios de gestión de "Artículos" y "Pedidos de Venta", con los campos de Tono, Calibre y Calidad incorporados, de forma que se puedan gestionar esta información.

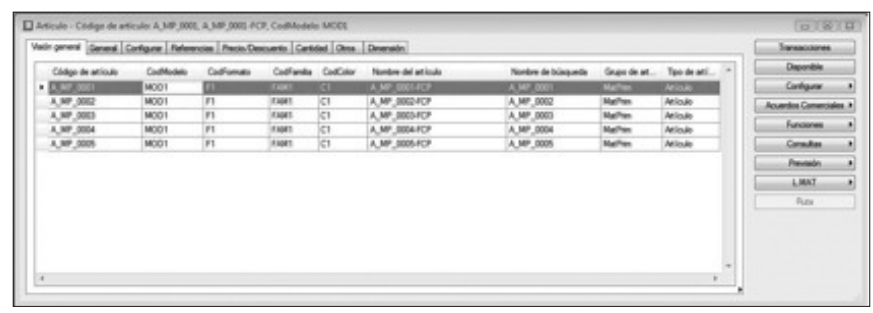

Figura 5. Formulario de Artículos adaptado a la gestión de TCC.

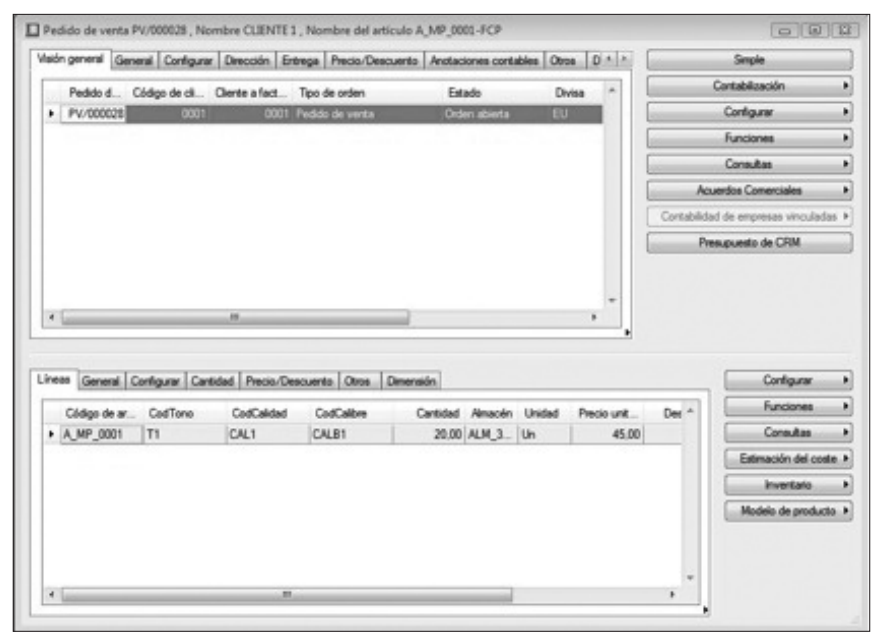

Figura 6. Formulario de Pedidos de Venta adaptados a la gestión del TCC. 


\section{CONCLUSIONES}

En el presente artículo se justifica la necesidad de desarrollar soluciones ERP verticales adaptadas al sector cerámico. Se han analizado los requerimientos que dicho sector presenta en cuanto a la identificación de los productos fabricados, derivados de la problemática generada por la FHP.

En base a dichos requerimientos, se ha diseñado un modelo de datos para solucionar los problemas de identificación de producto que la FHP genera en el sector cerámico. Éste modelo de datos debería ser incorporado en los sistemas ERP verticales para el sector cerámico. Queda pendiente la integración del modelo de datos propuesto a través de todos los procesos de la empresa, tarea que deberá ser realizada por los desarrolladores, y que depende de la plataforma de desarrollo que utiliza cada ERP.

Sin embargo, sí que es posible, y necesario, ofrecer a los desarrolladores una guía para la adaptación del modelo de datos en los diferentes sistemas ERP. En este artículo se ha presentado a modo de ejemplo la integración del modelo de datos propuesto con las tablas del pedido de venta y del inventario. Este ejemplo, muestra a los desarrolladores de sistemas ERP, cómo deberían incorporar el modelo de datos de identificación del artículo propuesto para el sector cerámico, a través de todos los procesos de la empresa, y por tanto, de todas las tablas, formularios y consultas del ERP, con el objetivo de desarrollar en una solución vertical para el sector cerámico. Para ello, se ha desarrollado el modelo ER y una pequeña base de datos en Access, que se ha trasladado en una aplicación práctica al ERP Dynamics AX.

Como continuación de este trabajo, cabe destacar que una vez el modelo de datos haya sido incorporado en el ERP, ya se puede disponer de la información necesaria, y se pueden diseñar e incorporar herramientas de ayuda a la toma de decisiones que optimicen los procesos de la empresa en los que influye la FHP. Esto, hasta ahora, sin la identificación adecuada de los productos no era posible.

De esta forma, tras este trabajo, surgen diferentes líneas de investigación, como son el desarrollo de herramientas de optimización y de ayuda a la toma de decisiones. Por ejemplo, un modelo matemático que optimice la asignación de inventario a pedidos de venta, modelos de cálculo de previsiones que tengan en cuenta las calidades, tono y calibres, o el diseño de un modelo de datos que sea capaz de gestionar órdenes de producción en los que un input de lugar a diferentes outputs.

\section{BIBLIOGRAFÍA}

(1) M. D. Arango, H. Gil, y R. F. Oltra, «Evolutions and Trends of Information Systems for Business Management: the M-Business. A Review», Dyna, vol. $77, \mathrm{n}^{\circ} .163$, págs. $110-125,(2010)$.

(2) B. Grabot y V. Botta-Genoulaz «Special issue on Enterprise Resource Planning (ERP) systems», Comput. Ind., vol. 56, nº. 6, págs. 507-509, (2005).

(3) S. Trimi, S. M. Lee, D. L. Olson, y J. Erickson, "Alternative means to implement ERP: Internal and ASP», Ind. Manage. Data. Syst., vol. 105, nº. 2, págs. 184 - 192, (2005).

(4) L. T. Ho, G. Lin, y S. Nagalingam, «A risk mitigation framework for integrated-enterprise systems implementation for the manufacturing environment», Int. J. Bus. Inform. Syst., vol. 4, págs. 290-310, (2009).

(5) J. Albors, P. Márquez, y J. L. Hervás, «Las relaciones fabricante distribuidor como elementos básicos de un modelo competitivo en el caso del cluster cerámico español: .Análisis empírico de los factores moderadores», Bol. Soc. Esp. Ceram. Vidrio, vol. 47, nº. 6, págs. 339 -344, (2008).
(6) J. Albors, P. Márquez, y M. Segarra, «Internet como herramienta de creación de valor en sectores maduros. El caso de los productores y distribuidores cerámicos en España», Bol. Soc. Esp. Ceram. Vidrio, vol. 48, n. 6, págs. 279-288, (2009).

(7) E. Vallada, C. Maroto, R. Ruiz, y B. Segura, «Problemas de programación de la producción en el sector cerámico español», presented at the 27 Congreso Nacional de Estadística e Investigación Operativa, Lleida España, págs. 638-676, (2003).

(8) E. Vallada, C. Maroto, R. Ruiz, y B. Segura, «Análisis de la programación de la producción en el sector cerámico español», Bol. Soc. Esp. Ceram. Vidrio, vol. 44, nº. 1, págs. 39 - 44, (2005).

(9) F. Alarcón, M. del M. E. Alemany, F. C. Lario, y R. F. Oltra, «La falta de homogenidad del producto (FHP) en las empresas cerámicas y su impacto en la reasignación del inventario», Bol. Soc. Esp. Ceram. Vidrio, vol. 50, nº. 1, págs. 49-58, (2011).

(10) R. Oltra-Badenes "Identificación de tendencias de los sistemas integrados de gestión empresarial. Análisis funcional y diseño de herramientas para el desarrollo de un ERP vertical adaptado al sector cerámico". Doctoral Thesis, Universidad Politécnica de Valencia, Valencia, Spain. (2012)

(11) R. F. Oltra, H. Gil, y J. V. Oltra, «Evolución y tendencias de los sistemas de informacion para la gestión en la empresa: el m-commerce», presented at the VI CONGRESO SOCOTE-I Congreso Iberoamericano SOCOTE, Valencia, págs. 212 - 230, (2009).

(12) R. Kohavi, N. J. Rothleder, y E. Simoudis, «Emerging trends in business analytics», Commun. ACM, vol. 45, nº. 8, págs. 45-48, (2002).

(13) J. Albors, J. L. Hervas, y P. Marquez, «Adopción contingente de tecnología de producción en el sector cerámico español. Un estudio empírico.», Bol. Soc. Esp. Ceram. Vidrio, vol. 45, nº. 5, págs. 338 - 345, (2006).

(14) J. Albors y J. L. Hervas, «La Industria cerámica europea en el siglo XXI Retos tecnológicos y desafios de la próxima década», Bol. Soc. Esp. Ceram. Vidrio, vol. $45, \mathrm{n}^{\circ} .1$, págs. $13-21,(2006)$.

(15) R. Baldrich, M. Vanrell, y J. J. Villanueva, «Polarization and Color Techniques in Industrial Inspection», presented at the Polarization and Color Techniques in Industrial Inspection, Munich, Germany, vol. 3826, págs. 124-135, (1999).

(16) S. Kukkonen, H. Kalviainen, y J. Parkkinen, «Color features for quality control in ceramic tile industry», Opt. Eng., vol. 40, nº. 2, págs. 170-177, (2001).

(17) R. Ruiz y C. Maroto, "A genetic algorithm for hybrid flowshops with sequence dependent setup times and machine eligibility», Eur. J. Oper. Res., vol. $169, \mathrm{n}^{\circ} .3$, págs. 781-800, (2006).

(18) I. Tortajada, G. Peris-Fajarnes, M. Aguilar, y P. Latorre, «Análisis del proceso de clasificación cerámica», Bol. Soc. Esp. Ceram. Vidrio, vol. 45, nº. 1 , págs. $22-27,(2006)$.

(19) J. A. Heredia y M. Gras, «Análisis y modelado de la transmisión de variabilidaddimensional en un proceso de producción de baldosas cerámicas», Bol. Soc. Esp. Ceram. Vidrio, vol. 48, no. 6, págs. 289-296, (2009).

(20) L. Fink y S. Markovich, «Generic verticalization strategies in enterprise system markets: An exploratory framework», J. Inform. Tech., vol. 23, págs. 281-296, (2008)

(21) T. F. Gattiker y D. L. Goodhue, «Understanding the local-level costs and benefits of ERP through organizational information processing theory», Inform. Manage., vol. 41, nº. 4, págs. 431-443, (2004).

(22) A. Jakupović, M. Pavlic, y P. Poscic, «Business sectors and ERP solutions», in Information Technology Interfaces (ITI), 2010 32nd International, Cavtat/ Dubrovnik, págs. 477-482, (2010).

(23) C. Ferran y R. Salim, Enterprise Resource Planning for Global Economies: Managerial Issues and Challenges NetLibrary, Inc Premier Reference Source. Idea Group Inc (IGI), (2008).

(24) A. Jakupović, M. Pavlic, y M. Asenbrener, «Measuring the Size and Contribution of ERP Solutions through Covered Business Sectors», in ISAS Information Systems Analysis Synthesis, Orlando, Florida, (2010).

(25) M. Bertolini, M. Bevilacqua, E. Bottani, y A. Rizzi, «Requirements of an ERP enterprise modeller for optimally managing the fashion industry supply chain», J. Enterp. Inform. Manage., vol. 17, págs. 180-190, (2004).

(26) M. Bergström y L. Stehn, «Matching industrialised timber frame housing needs and enterprise resource planning: A change process», Int. J. Prod. Econ., vol. 97, nº. 2, págs. 172-184, (2005).

(27) C.C. Yang, W.T. Lin, M.Y. Lin, y J.T. Huang, «A study on applying FMEA to improving ERP introduction: An example of semiconductor related industries in Taiwan», Int. J. Qual. Reliab. Manage., vol. 23, págs. 298-322, (2006).

(28) C. J. Stefanou y A. Revanoglou, «ERP integration in a healthcare environment: a case study», J. Enterp. Inform. Manage., vol. 19, págs. 115130, (2006)

(29) N. Pollock y J. Cornford, «ERP systems and the university as a "unique" organisation», Inform. Tech. People, vol. 17, págs. 31-52, (2004).

(30) B. Zhou, S. Wang, y L. Xi, «Data model design for manufacturing execution system», J. Manuf. Tech. Manage., vol. 16, págs. 909-935, (2005).

Recibido: $24 / 05 / 2011$

Aceptado: 14/01/2013 